\section{Evolución del síndrome metabólico y de sus componentes en un seguimiento de 10 años en adultos de la Región de Valparaíso}

\author{
PATRICIA BUSTOS, HUGO AMIGOa, \\ ALEJANDRA VÁSQUEZ ${ }^{\mathrm{b}}$, CLAUDIO VARGAS
}

\section{Evolution of the metabolic syndrome and its components in a follow up of 10 years in adults from Valparaíso region}

Background: Metabolic syndrome is a risk factor for cardiovascular diseases and cancer. Its frequency is increasing steadily Aim: To evaluate the prevalence of metabolic syndrome (MS) and its components in a 10-year tracking study of young adults. Material and Methods: Concurrent cohort study of subjects born between 1974 and 1978. They were evaluated between 2000 and 2002 and between 2010 and 2012 to determine the frequency of MS and its components (high blood glucose, waist circumference [WC], triglycerides, blood pressure and low HDL cholesterol), according to ATP III criteria. Attrition was handled using the reciprocal of the probability of remaining in the study. Result: During the first evaluation, the prevalence of metabolic syndrome was 9.3\%, confidence interval (CI): 7.5-11.1), with no gender differences. Ten years later, the prevalence of MS increased significantly to $27.6 \%$ (CI: 24.7-29.9) and was more common in women than men (30.4 and 23.8\% respectively, $p<0.014)$. The components of MS also increased from one period to another: hyperglycemia, from $5.2 \%$ (CI: 4-7) to $24.4 \%$ (CI: 22-27); high triglyceride levels from $17.6 \%$ (CI: $15-20$ ) to $35.3 \%$ (CI: 32-38); high blood pressure from 14.7 (CI: 13-18) to 30.2\% (IC: 28-33) and high WC: $16.9 \%$ (CI: 15-19) to 41.5\% (CI: 39-45). In both evaluations, there was a greater frequency of high triglycerides and high blood pressure among men, and greater frequency of low HDL and high WC among women. Hyperglycemia only showed differences by gender in the second measurement, and was greater among men. Conclusions: There was a marked increase in metabolic syndrome and its components in a 10-year interval, which is a warning sign of future cardiovascular risk.

(Rev Med Chile 2014; 142: 579-586)

Key words: Adult; Metabolic Syndrome X; Prevalence.

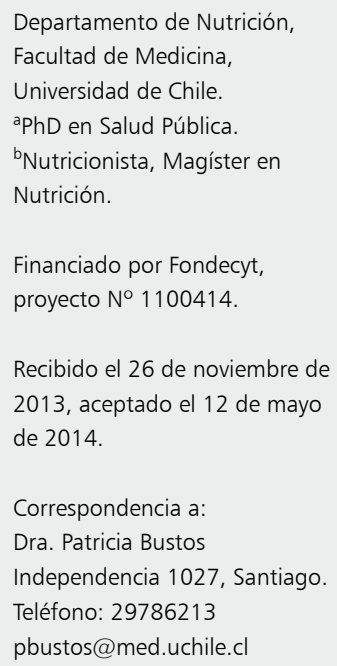

a mayoría de los países han tenido una
evolución favorable desde el punto de vista
de los problemas de salud que afectan a la
población infantil ${ }^{1}$, pero los cambios han sido des- favorables para los adultos al haber aumentado los factores de riesgo de enfermedad cardiovascular, patología que es la principal causa de muerte en los países con desarrollo más avanzado ${ }^{2,3}$. Si sumamos 
a esto el aumento de la esperanza de vida de las poblaciones, se fundamenta la preocupación que tienen los gobiernos en la prevención, manejo y control de los principales riesgos para reducir la carga de esta enfermedad, lo que podría reducir también el costo económico que significan para las personas y el Estado.

El síndrome metabólico y sus componentes han sido aceptados como factores de riesgo cardiovascular y de cáncer en sus presentaciones más comunes ${ }^{4,5}$, con una prevalencia que va en aumento. Se ha señalado que este incremento dependería de numerosos factores como la edad en que se ha medido (mayor en las personas de la tercera edad), el aumento del exceso de peso, el tipo y calidad de alimentación que las personas reciben y el sedentarismo, entre otros ${ }^{6-8}$.

Chile, que ha tenido una rápida transición epidemiológica y nutricional ${ }^{9}$, no está ajeno al aumento de los factores de riesgo cardiovascular que cada vez aparecen a edades más tempranas ${ }^{10}$. La Encuesta Nacional de Salud realizada en el país reportó para el 2010 una prevalencia de síndrome metabólico de $27,5 \%$ para personas entre los 25 y 44 años, mayor en varones ${ }^{11}$, sin embargo, se desconoce el comportamiento de esta enfermedad en localidades de tamaño medio que si bien cuentan con servicios de atención, gran parte de su población no acude a ellos o no sigue tratamiento por no percibir o valorar adecuadamente sus condiciones de riesgo o porque las realidades laborales les dificultan acudir a control con la frecuencia que lo requieren.

Este estudio corresponde a un seguimiento de personas que nacieron en el Hospital de Limache, Región de Valparaíso. El Hospital de Limache es un centro asistencial que atiende a las comunas de Limache y Olmué, que contaban en el año 2012 con una población proyectada a partir del censo de 2002 , de 45.864 y 16.243 personas respectivamente $^{12}$ y cuyas principales fuentes de ingresos son las actividades agrícolas y más recientemente, el turismo. Estas comunas fueron elegidas por su baja tasa de migración ${ }^{13}$ y porque existe una red asistencial en salud de amplia cobertura que ha servido de base para la organización de este estudio.

El objetivo del presente trabajo fue evaluar la evolución del síndrome metabólico y sus componentes en dos períodos, con un intervalo de 10 años en la población de adultos nacidos en el Hospital de Limache entre los años 1974-1978.

\section{Material y Métodos}

Estudio de cohorte concurrente de personas nacidas en el Hospital de Limache, entre 1974 y 1978 las que fueron evaluadas en una primera oportunidad entre los años 2000-2002 y después, entre los años 2010-2012. En la primera evaluación, hubo una selección aleatoria $(\mathrm{n}=998$ personas) desde el listado de los nacidos vivos en el hospital. En la segunda evaluación, 37\% de la muestra original no se evaluó debido a pérdidas en el seguimiento, que correspondieron a personas que han emigrado (166), direcciones inubicables (116), ubicados pero no contactados después de múltiples visitas (115), rehusaron participar (29), muertes (6). Detalles de la composición de esta cohorte se pueden observar en una publicación reciente $^{14}$. Para el manejo de las pérdidas del segundo análisis, y para resguardar la aleatoriedad de la muestra inicial, se ocupó un factor de ponderación en que se utilizó el recíproco de la probabilidad de las pérdidas considerando las diferencias entre participantes y no participantes, de acuerdo a los procedimientos señalados por Weuve y cols ${ }^{15}$.

En ambas oportunidades, los participantes fueron entrevistados y evaluados antropométricamente en el hospital local o eventualmente en sus domicilios, por enfermeras o nutricionistas, capacitadas y permanentemente supervisadas.

Las mediciones que se realizaron fueron: perímetro de cintura, el que se obtuvo a través de mediciones efectuadas con una cinta flexible, en el punto medio entre la última costilla y la cresta iliaca. La variable se trabajó categorizada en valores normales y elevados (cifras $\geq 88 \mathrm{~cm}$ en mujeres y $\geq 102 \mathrm{~cm}$ en hombres). Presión arterial sistólica y diastólica, la que se midió en reposo (al finalizar la encuesta de salud), en dos oportunidades, con un manómetro automático (Omrom 740). Se trabajó con el promedio de las dos mediciones, considerándose hipertensión valores $\geq 130 / 85 \mathrm{~mm} \mathrm{Hg}$. Se tomó además una muestra de sangre con ayuno de al menos $8 \mathrm{~h}$, la que después de centrifugada y obtenido el plasma o suero, se guardó congelada para la determinación de glicemia, triglicéridos y HDL en el Laboratorio de Nutrición de la Pontificia Universidad Católica de Chile. La glicemia se evaluó mediante método enzimático colorimétrico (GOD/PAD method, Human, Alemania), triglicéridos también con método enzimático (Gesellschaft für Biochemica und Diagnostica mbH, 
Alemania). Los HDL se evaluaron por el método de Siegler y Wu en la primera oportunidad y por método enzimático la segunda vez, esta técnica es preferida en la actualidad por ser más rápida y automatizable, lo que permite un mayor control a través de estándares y controles adecuados lo que redunda en una mejor precisión.

Los puntos de corte para considerar valores alterados de estas variables, al igual que para los dos anteriormente mencionados, son los que indica ATP III actualizado el $2005^{16}$ : glicemia elevada, $\geq 100 \mathrm{mg} / \mathrm{dL}$, triglicéridos altos, $\geq 150 \mathrm{mg} / \mathrm{dL}$ y HDL bajo, < 40 ó $50 \mathrm{mg} / \mathrm{dL}$ en hombres y mujeres respectivamente.

La información se digitó en el Programa EPIDAT y el análisis estadístico se realizó con el programa SPSS versión, 20,0.

Inicialmente se realizó un análisis de consistencia de los datos, revisando los valores fuera de rango y estudiando su distribución, luego, se describió la población en estudio a través de medidas centrales (mediana y percentiles 25 y 75), se calculó la prevalencia de los componentes del síndrome metabólico (SM), considerándose caso prevalente al que tenía 3 o más variables alteradas en cada evaluación.

Este estudio fue aprobado por el Comité de Ética de la Facultad de Medicina de la Universidad de Chile. Los participantes firmaron un consentimiento informado en ambas oportunidades antes de comenzar el estudio.

\section{Resultados}

Entre los participantes predominaron las mujeres (54,9\%), las medianas de edad en cada momento fueron homogéneas ya que dependen del diseño del estudio original: 25 años (IC: 24-26) en la primera evaluación y 35 años (IC: $34-37$ ) en la segunda, las medianas de escolaridad corresponden a enseñanza media completa, la que no se modificó entre un período y otro. Con respecto al estado nutricional, hubo escasa frecuencia de enflaquecimiento pero alta prevalencia de exceso de peso en los dos momentos analizados, con una disminución de las personas con estado nutricional normal para aumentar las con sobrepeso y obesidad en el seguimiento de 10 años. Según nivel socioeconómico (NSE), se constató que la población estudiada se concentró en ambas oportunidades en el nivel medio, aunque en la segunda evaluación, disminuyeron en al menos cuatro veces los pobres. En esta población no hubo personas clasificadas en NSE alto (Tabla 1).

Las medianas de todos los componentes del síndrome metabólico estuvieron en rangos normales en la primera evaluación, diez años después, las medianas aumentaron significativamente, especialmente los triglicéridos, aunque sólo las de perímetro de cintura superaron los valores recomendados en ambos sexos. Se comprobó que las medianas de los varones fueron superiores que las de las mujeres en los dos períodos, con excepción

Tabla 1. Características generales de la población estudiada en dos momentos: 2000-02 y 2010-12

\begin{tabular}{|c|c|c|c|}
\hline & Primera evaluación & Segunda evaluación & $\mathbf{p}$ \\
\hline Edad Med (EIC) & $25,0(24-26)$ & $35,3(34-37)$ & 0,0001 \\
\hline Escolaridad Med (EIC) & $12,0(9-12)$ & $12,0(9-12)$ & 0,0001 \\
\hline \multicolumn{4}{|l|}{ Estado nutricional (\%) } \\
\hline Enflaquecidos & $1,1(0,5-1,6)$ & $0,3(0,02-1,7)$ & \\
\hline Normales & $49,7(46,8-52,4)$ & $24,9(22,6-27,5)$ & 0,0001 \\
\hline Sobrepeso & $35,2(32,5-37,9)$ & $42,8(39,6-45,1)$ & \\
\hline Obesos & $14,0(12,1-15,9)$ & $31,9(29,6-34,9)$ & \\
\hline \multicolumn{4}{|c|}{ Nivel socioeconómico (\%) } \\
\hline Medio & $75,6(72,8-78,4)$ & $94,6(93,3-95,8)$ & \\
\hline Bajo & $24,4(22-26,8)$ & $5,4(4,1-6,7)$ & 0,001 \\
\hline
\end{tabular}

Med $=$ Mediana. $\mathrm{EIC}=$ Espacio inter cuartil. 


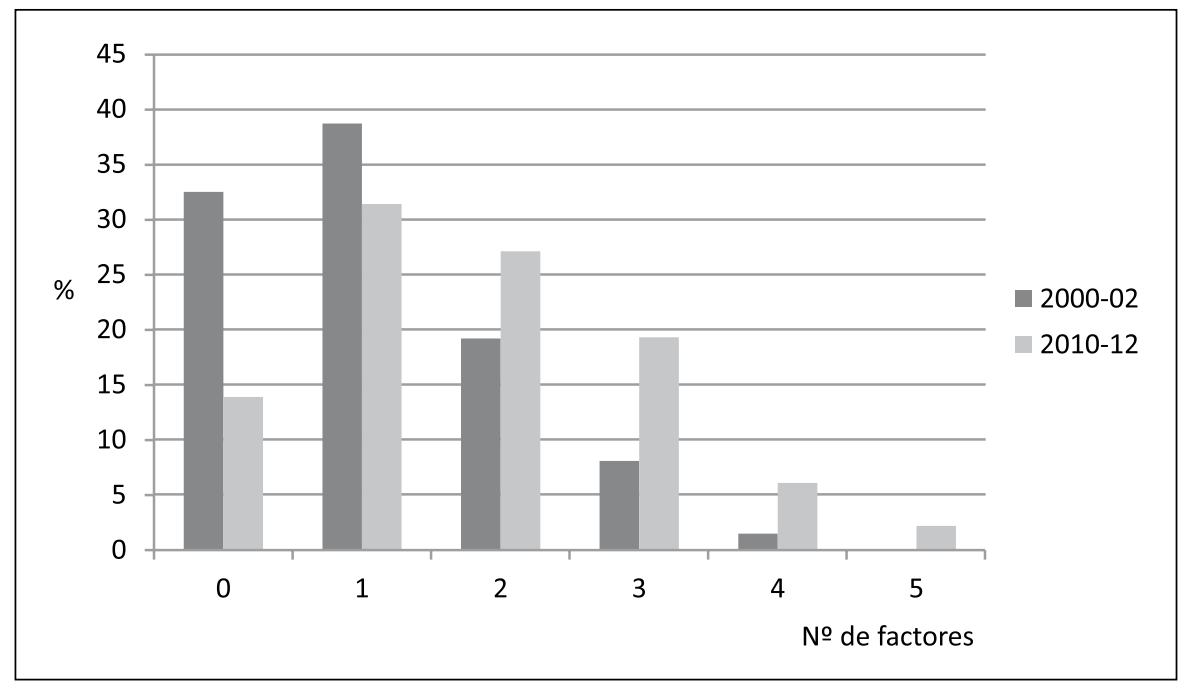

Figura 1. Prevalencia de factores de riesgo cardiovascular en los dos períodos estudiados. de los HDL cuyas medianas fueron mayores en las mujeres en la primera evaluación y sin diferencias por sexo en la segunda evaluación. También se observa el aumento antes descrito de un período a otro, en ambos sexos, con excepción de los HDL en mujeres en las que no se observaron cambios (Tabla 2).

Las prevalencias de cada uno de los componentes del síndrome metabólico también aumentaron de un período al otro: casi 5 veces para la glicemia elevada, al doble en triglicéridos y presión arterial elevada y casi 3 veces para perímetro de cintura elevado. Los HDL bajos disminuyeron, descenso que se constató sólo en los varones ya que en las mujeres las frecuencias se mantuvieron. En ambas evaluaciones, se observaron mayores cifras de triglicéridos altos y presión sistólica y diastólica elevadas en hombres, mientras que las frecuencias de HDL bajos y perímetro de cintura elevado fueron mayores en mujeres. La prevalencia de glicemia $\geq$ $100 \mathrm{mg} / \mathrm{dL}$ no se diferenció por sexo en la primera evaluación mientras que en la segunda medición, fue mayor en varones (Tabla 3 ).

En la primera oportunidad, 32,5\% de las personas no tenían factores de riesgo (IC: 27,4-37,6), porcentaje que se reduce a $14,6 \%$ (IC: $9,3-19,9)$ en la segunda evaluación. Treinta y nueve porciento (IC: 34,2-43,8) tenían un factor de riesgo cifra que se redujo a $31,3 \%$ (IC: $28,9-33,7$ ) en la segunda medición. Por otro lado, la sumatoria de 4 ó 5 factores de riesgo era prácticamente inexistente en la primera medición, mientras que en la segunda alcanza, en conjunto a 8\% (Figura 1).

El síndrome metabólico comprometió a 9,3\% de las personas estudiadas (IC: 7,5-11,1) en la primera evaluación y 27,6\% (IC: 24,7-29,9), 10 años después $(\mathrm{p}<0,0001)$. En la primera medición no hubo diferencias por sexo mientras que en la segunda evaluación, fue más frecuente en las mujeres: $30,4 \%$ (IC: $26-33$ ) versus $23,8 \%$ (IC: 21-28) en varones, $\mathrm{p}<0,014$.

\section{Discusión}

En este trabajo, efectuado en personas evaluadas con un intervalo de 10 años, se encontró un aumento significativo del síndrome metabólico y de casi todos sus componentes. El mayor aumento correspondió a los valores elevados de glicemia, seguidos por el aumento de las personas con perímetro de cintura elevado. Destaca también la particularidad de presentación de algunos componentes más frecuentes en varones (triglicéridos y presión arterial elevados) y otros en mujeres (perímetro de cintura elevado y HDL bajos).

Es esperable que a medida que pasan los años en la edad adulta aumenten las medianas de los factores de riesgo cardiovascular, el $\mathrm{SM}^{17}$ y varios de sus componentes. Esto se ha descrito especialmente en mujeres, en la menopausia ${ }^{18,19}$. En nuestro estudio, las cifras de SM aumentaron en 

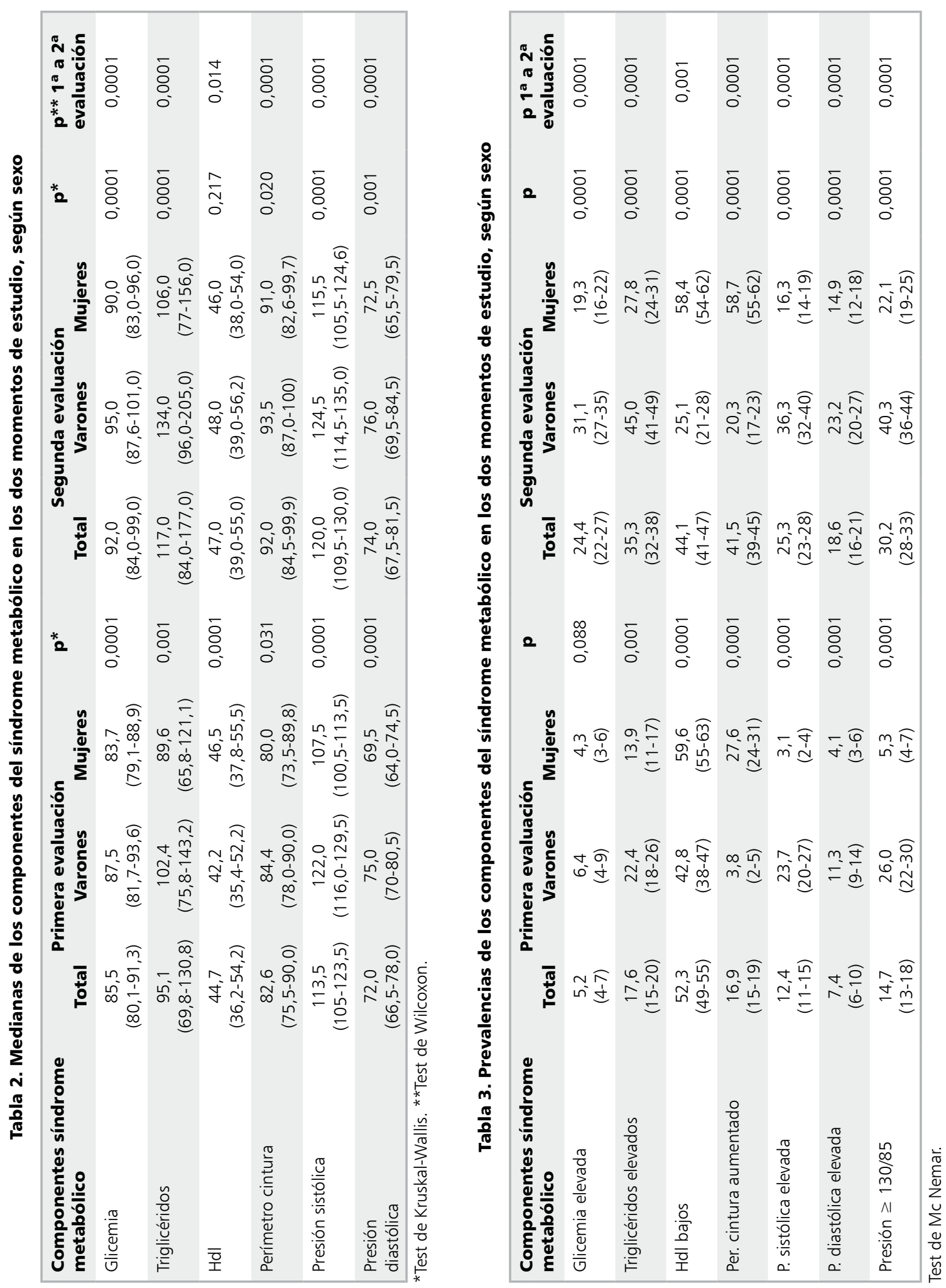
forma marcada, a pesar de que las personas estudiadas están lejos de la tercera edad. Cabe destacar que si se realizara un tratamiento adecuado, es posible que estas cifras se estabilizaran o bajaran como ha sido reportado en Estados Unidos de Norteamérica en que la prevalencia de SM bajó de 25,5 a 22,9\%, indicándose que los componentes que mejoraron en las personas tratadas fueron los lípidos y la presión arterial elevados ${ }^{20}$.

El haber encontrado mayor prevalencia de síndrome metabólico en mujeres en la segunda evaluación es contrario a lo descrito en la mayoría de las publicaciones ${ }^{21,22}$, aunque hay estudios que han reportado también mayor frecuencia en mujeres ${ }^{17,23}$, lo que podría deberse a la alta frecuencia de valores elevados de perímetro de cintura en ellas ${ }^{24,25}$. El aumento del perímetro de cintura en nuestro estudio es coherente con el aumento de las cifras de obesidad que cambiaron de $14 \%$ en la evaluación de $2000-02$ a $32 \%$ en el 2010-12. Es preciso señalar que en nuestro estudio se usaron puntos de corte para los componentes del SM recomendados por ATP III, actualizados el año $2005^{16}$, ya que en nuestro país aún no hay consenso para el cambio de valores para el PC. Diversas publicaciones recientes indican la necesidad de utilizar puntos de corte según etnia para esta medición, ya que en población asiática, algunas personas tienen resistencia a la insulina al aumentar levemente su PC, por lo que en ellos se usa un punto de corte menor ( 80 en mujeres, 94 en hombres) $)^{26,27}$.

Los valores altos de las variables estudiadas, especialmente de obesidad abdominal, pueden deberse a diversos factores como sedentarismo, ingesta superior a las recomendaciones y también a un efecto del nivel socioeconómico en la población, ya que se ha descrito que hay una relación inversa entre la prevalencia de obesidad con $\mathrm{NSE}^{28}$ y de síndrome metabólico con $\mathrm{NSE}^{29}$. La relevancia de estos valores elevados en población relativamente joven es por su asociación con infarto al miocardio a edades tempranas ${ }^{4,30}$.

Contrario a lo esperado, la prevalencia de HDL bajos disminuyó en hombres, esta situación podría estar relacionada con el cambio de técnica utilizada para la determinación de estas lipoproteínas, aspecto que está determinando también que la prevalencia de SM no sea algo más alta que la comunicada. El que la prevalencia de HDL bajos sea mayor en mujeres pareciera esperable debido al elevado punto de corte que se usa en ellas para identificar los valores bajos o alterados.

Entre las debilidades de este estudio está el haber perdido algunos casos, aunque se debe mencionar que en todos los estudios de seguimiento hay personas que no es posible volver a evaluar y era esperable tener pérdidas en este seguimiento de 10 años. Considerando este factor, las pérdidas fueron tratadas por el método para corregir los sesgos provenientes de las pérdidas usando el inverso de la probabilidad de permanencia en el estudio, esto es aquel con menos probabilidad de haber permanecido en el estudio se pondera más que aquel que tienen alta probabilidad de permanencia ${ }^{15}$.

Entre las fortalezas está el que se trata de una población numerosa de comunas con un porcentaje considerable de población semirural. Estas personas fueron entrevistadas y evaluadas por personal capacitado y permanentemente supervisado.

En este estudio se pueden percibir las consecuencias de la rápida transición epidemiológica y nutricional que ha vivido el país en las últimas décadas y reflejadas a nivel nacional por ejemplo, con el aumento de las cifras de obesidad de la población adulta (de $23,2 \%$ a $27,4 \%$ en las encuestas nacionales de salud realizadas en el país los años 2003 y 2009) o de diabetes tipo 2 (de $6,3 \%$ a $9,4 \%)^{11}$ y objetivadas a nivel local en el caso de nuestro. Esta cohorte de Limache refleja un interesante seguimiento, al tratarse de adultos jóvenes de la comunidad, en una edad en la que hay pocos estudios de cohorte y de personas que no están incorporadas en programas de seguimiento y control, por lo que nos parece que este tipo de estudios son muy necesarios al permitir entregar antecedentes para intervenciones de una población poco investigada.

\section{Referencias}

1. UNICEF. Estado Mundial de la Infancia, Informe anual. 2012 [updated 2012; cited 2013 octubre, 2013]; Available from: www.unicef.org.

2. OMS. Informe sobre la salud en el mundo. 2013 [updated 2013; cited 2013 octubre 2013]; Available from: www.who.int/publications/en.

3. Lozano R, Naghavi M, Foreman K, Lim S, Shibuya K, Aboyans $\mathrm{V}$, et al. Global and regional mortality from 235 causes of death for 20 age groups in 1990 and 2010: 
a systematic analysis for the Global Burden of Disease Study 2010. Lancet 2012; 380 (9859): 2095-128.

4. Schmidt C, Bergstrom GM. The metabolic syndrome predicts cardiovascular events: results of a 13-year follow-up in initially healthy 58-year-old men. Metab Syndr Relat Disord 2012; 10 (6): 394-9.

5. Esposito K, Chiodini P, Colao A, Lenzi A, Giugliano D. Metabolic syndrome and risk of cancer: a systematic review and meta-analysis. Diabetes Care 2012; 35 (11): 2402-11.

6. Castanho GK, Marsola FC, McLellan KC, Nicola M, Moreto F, Burini RC. [Consumption of fruit and vegetables associated with the Metabolic Syndrome and its components in an adult population sample]. Cien Saude Colet 2013; 18 (2): 385-92.

7. Shuval K, Finley CE, Chartier KG, Balasubramanian BA, Gabriel KP, Barlow CE. Cardiorespiratory fitness, alcohol intake, and metabolic syndrome incidence in men. Med Sci Sports Exerc 2012; 44 (11): 2125-31.

8. Sumner AD, Sardi GL, Reed JF, 3rd. Components of the metabolic syndrome differ between young and old adults in the US population. J Clin Hypertens (Greenwich) 2012; 14 (8): 502-6.

9. Vio F, Albala C, Kain J. Nutrition transition in Chile revisited: mid-term evaluation of obesity goals for the period 2000-2010. Public Health Nutr 2008; 11 (4): 40512.

10. Burrows R, Atalah E, Leiva L, Rojas P, de la Maza MP, Vasquez F, et al. [Metabolic syndrome prevalence in Chilean children and adolescent with family history of chronic noncommunicable diseases]. Arch Latinoam Nutr 2012; 62 (2): 155-60.

11. Ministerio de Salud de Chile. Encuesta Nacional de Salud, Chile 2009. 2010 [updated 2010; cited 02/05/2013]; Available from: http://www.encuestasalud.cl.

12. Instituto Nacional de Estadísticas de Chile. Población total 2002 y proyectada 2012: Estadísticas Comunales, Limache y Olmué. 2012 [updated 2012; cited]; Available from: www.reportescomunales.bcn.cl/.

13. CEPAL. Migración interna en América Latina y el Caribe. División Administrativa Menor. Chile. 2002 [updated 2002; cited]; Available from: http://www.eclac. cl/celade/migracion/migracion_interna/.

14. Amigo H, Bustos P, Zumelzu E, Rona R. Cohort profile: The Limache, Chile, birth cohort study. International Journal of Epidemiology 2013; In Press. doi: 10.1093/ ije/djt091.

15. Weuve J, Tchetgen Tchetgen EJ, Glymour MM, Beck TL, Aggarwal NT, Wilson RS, et al. Accounting for bias due to selective attrition: the example of smoking and cognitive decline. Epidemiology 2012; 23 (1): 119-28.
16. Grundy SM, Cleeman JI, Daniels SR, Donato KA, Eckel RH, Franklin BA, et al. Diagnosis and management of the metabolic syndrome: an American Heart Association/National Heart, Lung, and Blood Institute Scientific Statement. Circulation 2005; 112 (17): 2735 52.

17. Xi B, He D, Hu Y, Zhou D. Prevalence of metabolic syndrome and its influencing factors among the Chinese adults: The China Health and Nutrition Survey in 2009. Prev Med 2013; 57 (6): 867-71.

18. Chedraui P, San Miguel G, Vintimilla-Siguenza I, Villacreses D, Romero-Huete L, Dominguez A, et al. The metabolic syndrome and its components in postmenopausal women. Gynecol Endocrinol 2013; 29 (6): 563-8.

19. Jouyandeh Z, Nayebzadeh F, Qorbani M, Asadi M. Metabolic syndrome and menopause. J Diabetes Metab Disord 2013; 12 (1): 1.

20. Beltrán-Sánchez H, Harhay MO, Harhay MM, McElligott S. Prevalence and trends of metabolic syndrome in the adult US Population, 1999-2010. J Am Coll Cardiol 2013 Aug 20; 62 (8): 697-703.

21. Ilow R, Regulska-Ilow B, Rozanska D, Kowalisko A, Biernat J. Prevalence of metabolic syndrome among 40- and 50 -year-old inhabitants of Wroclaw, Poland. Ann Agric Environ Med 2012; 19 (3): 551-6.

22. Hadaegh F, Hasheminia M, Lotfaliany M, Mohebi R, Azizi F, Tohidi M. Incidence of Metabolic Syndrome over 9 Years Follow-Up; the Importance of Sex Differences in the Role of Insulin Resistance and Other Risk Factors. PLoS One 2013; 8 (9): e76304.

23. Erem C, Hacihasanoglu A, Deger O, Topbas M, Hosver I, Ersoz HO, et al. Prevalence of metabolic syndrome and associated risk factors among Turkish adults: Trabzon MetS study. Endocrine 2008; 33 (1): 9-20.

24. Oguz A, Temizhan A, Abaci A, Kozan O, Erol C, Ongen $\mathrm{Z}$, et al. Obesity and abdominal obesity; an alarming challenge for cardio-metabolic risk in Turkish adults. Anadolu Kardiyol Derg 2008; 8 (6): 401-6.

25. Ghandehari H, Le V, Kamal-Bahl S, Bassin SL, Wong ND. Abdominal obesity and the spectrum of global cardiometabolic risks in US adults. Int J Obes (Lond) 2009; 33 (2): 239-48.

26. Kamezaki F, Sonoda S, Nakata S, Kashiyama K, Muraoka Y, Okazaki M, et al. Proposed cutoff level of waist circumference in Japanese men: evaluation by homeostasis model assessment of insulin resistance levels. Intern Med 2012; 51 (16): 2119-24.

27. Oka R, Yagi K, Sakurai M, Nakamura K, Nagasawa SY, Miyamoto S, et al. Impact of visceral adipose tissue and 
Síndrome metabólico: seguimiento de 10 años en adultos de la Región de Valparaíso - P. Bustos et al

subcutaneous adipose tissue on insulin resistance in middle-aged Japanese. J Atheroscler Thromb 2012; 19 (9): 814-22.

28. Drewnowski A, Moudon AV, Jiao J, Aggarwal A, Charreire $\mathrm{H}$, Chaix B. Food environment and socioeconomic status influence obesity rates in Seattle and in Paris. Int J Obes (Lond) 2013 May 27.

29. Zhan Y, Yu J, Chen R, Gao J, Ding R, Fu Y, et al. Socioe- conomic status and metabolic syndrome in the general population of China: a cross-sectional study. BMC Public Health 2012; 12: 921-7.

30. Kazemi T, Sharifzadeh G, Zarban A, Fesharakinia A. Comparison of components of metabolic syndrome in premature myocardial infarction in an Iranian population: a case -control study. Int J Prev Med 2013; 4 (1): $110-4$. 